\title{
Introduction to the Minitrack
}

\section{Mobile Apps and Wearables for Health Management, Analytics, and Decision Making}

\author{
Prof. Dr. Freimut Bodendorf \\ University of Erlangen- \\ Nuremberg, Germany \\ freimut.bodendorf@,fau.de
}

\author{
Prof. Nilmini Wickramasinghe \\ Epworth HealthCare and \\ Deakin University, Australia \\ nilmini.work@gmail.com
}

\author{
Tuan Huy Ma \\ Fraunhofer Institute IIS \\ Erlangen, Germany \\ tuan-huy.ma@scs.fraunhofer.de
}

Mobile Apps, wearables, and Social Media are dramatically influencing how clinicians, caregivers, patients and their families can manage health and wellness. Further, they have the potential to facilitate the design and development of superior healthcare delivery. In addition, there is a trend to foster active patient participation in their care and we are witnessing a plethora of emerging technologies to assist in this regard. The data collected enable sophisticated analysis and smart decision making. In this minitrack, we focus on how such technologies might be utilized to address the challenges faced by healthcare management such as escalating cost pressures, a growing aging population, an increasing prevalence of chronic diseases, and a move to a preventive care focus. Integral to these solutions is a patient-centric view in order to satisfy consumer expectations and provide high quality care.
The minitrack comprises two sessions. A set of presentations focuses on conceptual solutions as well as application scenarios of wearable and mobile devices for health and fitness. The physician's perspective as well as the patient-centric view will be addressed. Exemplary prototypical systems will be introduced. Another group of papers exhibits results of empirical studies conducted in clinical and ambient environments. In addition, the fast growing area of big data research is taken into account, looking at interesting methods and practices of descriptive, predictive, and prescriptive analytics. All together the contributions serve to illustrate the potentials and limits of mobile applications for health management and wellness.

For four years now the minitrack is home to a persistent and growing community of academic researchers and healthcare professionals who are interested in the potentials of mobile technologies and services. Future topics will include the Internet of Things and the coaction of persons, mobile devices, and autonomous systems. 